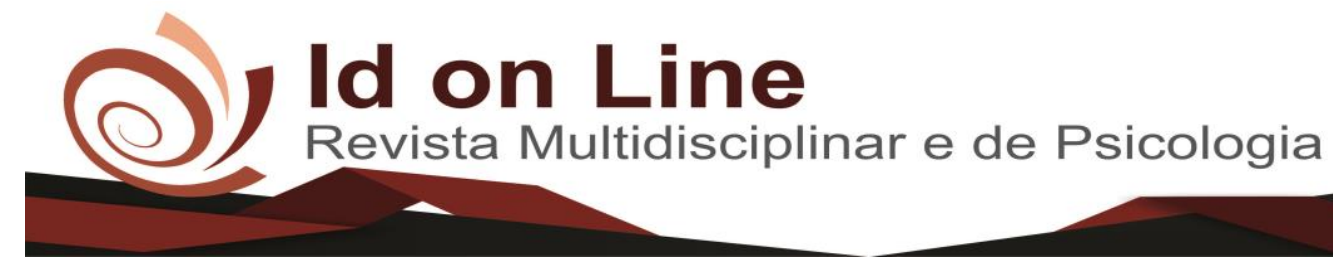

DOI: $10.14295 /$ idonline.v13i48.2247

Artigo

\title{
Avaliação Respiratória em Crianças com Síndrome de Down Submetidas a Treino Psicomotor
}

\author{
Eduarda Stefany Silva e Silva ${ }^{1}$; Laisla Pires Dutra ${ }^{2}$; \\ Aline Marques Piloto ${ }^{3}$; Daiane Santos Dias Saraiva
}

\begin{abstract}
Resumo: Os indivíduos com síndrome de Down apresentam uma pré-disposição a complicações respiratórias, decorrente não só da hipotonia generalizada, mas também de doenças infecciosas das vias aéreas inferiores e de obstrução de vias aéreas superiores. Dentre as características da síndrome, a hipotonia muscular e o déficit respiratório são as que mais requerem atenção, o que acaba contribuindo para os atrasos de desenvolvimento motor. $\mathrm{O}$ atual estudo tem como objetivo avaliar a eficácia do treino de psicomotricidade na capacidade respiratória de crianças com diagnóstico de síndrome de Down. Trata-se de um estudo quantitativo, de corte transverso e descritivo, onde foram incluídos no estudo crianças com faixa etária de 7 a 12 anos, de ambos os sexos, credenciadas a uma associação de pais e amigos de pessoas com síndrome de Down, no qual foram avaliadas a capacidade inspiratória máxima e capacidade expiratória máxima pré e pós a realização do treino psicomotor através de circuitos motores. Os resultados deste estudo sugerem que a prática de treino pode influenciar a força muscular respiratória em pacientes com síndrome de Down, de ambos os sexos, quando comparados com indivíduos com síndrome de Down que não praticam algum tipo de exercício.
\end{abstract}

Palavras-Chave : Crianças . Pressão Expiratória Máxima . Pressão Inspiratória Máxima . Síndrome de Down.

\section{Respiratory Assessment in Children with Down Syndrome Undergoing Psychomotor Training}

\begin{abstract}
Individuals with Down syndrome have a predisposition to respiratory complications , resulting not only from generalized hypotonia , but also from infectious diseases of the lower airways and upper airway obstruction. Among the characteristics of the syndrome, muscle hypotonia and respiratory deficit are the ones that most require attention, which ends up contributing to motor development delays. The present study aims to evaluate the effectiveness of psychomotor training on respiratory capacity in children diagnosed with Down syndrome. This is a quantitative, cross sectional and descriptive study, which included children aged 7 to 12 years, of both sexes, accredited to an association of parents and friends of people with Down syndrome, in the study. which were evaluated the maximum inspiratory capacity and maximum expiratory capacity before and after the accomplishment of the psychomotor training through motor circuits. The results of this study suggest that training may influence respiratory muscle strength in Down syndrome patients of both sexes when compared with individuals with Down syndrome who do not exercise.
\end{abstract}

Keywords: Children. Maximum Expiratory Pressure. Maximum Inspiratory Pressure. Down's Syndrome.

\footnotetext{
${ }^{1}$ Graduanda em Fisioterapia pela Faculdade Independente do Nordeste (FAINOR). eduardafisio23@hotmail.com;

${ }^{2}$ Professora Orientadora pela Faculdade Independente do Nordeste (FAINOR). laysla19@ hotmail.com;

${ }^{3}$ Graduanda em Fisioterapia pela Faculdade Independente do Nordeste (FAINOR). lynepiloto@ hotmail.com;

${ }^{4}$ Graduanda em Fisioterapia pela Faculdade Independente do Nordeste (FAINOR). daydd21@ hotmail.com.
} 


\section{Introdução}

A síndrome de Down (SD) foi descoberta por Jhon Langdon Haydon Down e foi reconhecida em 1866. Desde então, estudos realizados esclareceram ainda mais sobre a síndrome. Indivíduos com SD apresentam um cromossomo a mais no par 21; assim, a célula terá 47 cromossomos ao invés de 46, tornando assim uma célula diferente da convencional. A alteração da síndrome ocorre na formação do gameta ou após a fecundação, onde há uma separação inadequada do cromossomo 21, gerando variável grau de retardo no desenvolvimento físico, motor e mental, possuindo assim características únicas (GORLA; DUARTE; COSTA, 2017).

A SD apresenta mais de um tipo de alteração celular, sendo elas de três tipos: Trissomia livre do cromossomo 21, a mais comum, mosaiscismo cromossômico e por translocação entre os cromossomos. Pesquisadores ainda não descobriram o mecanismo da SD, mas sabe-se que o material genético extra, apresenta um desequilíbrio genético, fazendo com que a célula cresça e desenvolva incompleta e não anormal (MARINHO, 2018).

O fenótipo da SD é complexo e varia de acordo com cada pessoa, podendo assim ter várias características; as mais comuns são a hipotonia generalizada, frouxidão ligamentar, força muscular diminuída, desgastes nas articulações e problemas respiratórios; dando destaque aos problemas respiratórios, sendo a maior causa de internação e morte nesta população, devido à obstrução de vias aéreas superiores e doenças de vias aéreas inferiores, podendo ser ou não acompanhadas de uma cardiopatia congênita (LIMA et al.,2018).

A hipotonia muscular generalizada vai fazer com que o diafragma fique fraco em consequência da própria postura, baixo reflexo de tosse e grande produção de muco, sendo mais frequente a pneumonia obstrutiva considerada a maior causa de internamento em UTI, como complicação também a fadiga muscular, hipoplasia pulmonar, hipertensão pulmonar, entre outras (ALCÂNTARA, 2018).

A SD por ser uma patologia que aborda diferentes complicações, tem por sua vez o trabalho de uma equipe, sendo multidisciplinar ou interdisciplinar, trazendo 7 assim um papel muito importante para o desenvolvimento dessa criança em vários aspectos, melhorando a sua qualidade de vida. Dentre os profissionais da área da saúde que atuam nesse desenvolvimento, o fisioterapeuta ganha um grande destaque nessa patologia, sendo assim primordial para o 
desenvolvimento dessa criança, que pode ser estimulada desde os seus primeiros meses de vida (FALCÃO, 2018).

Com grande importância, a fisioterapia irá atuar no desenvolvimento motor, onde tem grande destaque seguido do trabalho cardiorrespiratório que irá melhorar muito a sua qualidade de vida. Dentre o trabalho motor, serão trabalhadas as habilidades da motricidade fina e grossa, o atraso motor, a prevenção de deformidades ósseas e a prevenção de instabilidades articulares. Junto a esse procedimento, será também trabalhada a parte cardiorrespiratória desses indivíduos, pois, fortalecendo a musculatura respiratória, irá melhorar muito a sua qualidade de vida e assim diminuir internações e complicações futuras (BRAGA, et al., 2019).

A psicomotricidade vem ganhando uma grande importância na fisioterapia, onde a criança é estimulada desde os primeiros meses de vida, ampliando a capacidade de se adaptar às necessidades comuns. Através do brincar, a criança começa a descobrir novas coisas, a ampliar o conhecimento, a entender seus sentimentos e a se adaptar ao meio em que vive. $\mathrm{O}$ brincar envolve a movimentação física e é fundamental para o processo intelectivo. O exercício físico tem uma grande importância para o desenvolvimento da criança, estimulando a circulação, o aparelho digestivo e a respiração; além de fortalecer músculos e melhorar a capacidade física (HASEGAWA, et al., 2018).

O uso do circuito de forma lúdica para o fortalecimento da musculatura global proporciona um melhoramento respiratório, melhor equilíbrio, o que irá contribuir de forma positiva para o desenvolvimento da criança. Sendo assim, o objetivo geral desse estudo é avaliar a capacidade pulmonar dessa população, após treino de psicomotricidade.

Diante dos pressupostos acima elencados, o presente estudo se propõe a responder a seguinte questão-problema: Quais os efeitos do treino psicomotor para a capacidade respiratória de crianças com Síndrome de Down?

Para tanto, a pesquisadora delimitou como objetivo geral avaliar os efeitos do treino psicomotor na capacidade respiratória de crianças com síndrome de Down. Como objetivos específicos: caracterizar o perfil sociodemográfico das crianças com síndrome de Down. verificar a PiMax e a PeMax antes e após o treino de psicomotricidade, descrever o planejamento das atividades propostas para os treinos de psicomotricidade realizadas com os participantes do estudo. 


\section{Método}

Trata-se de um estudo de intervenção, descritiva, exploratória e análise quantitativa. O estudo foi realizado na sede de uma associação de pais e amigos de pessoas com SD do município de Vitória da Conquista-Ba, sendo esta sociedade civil, sem fins lucrativos, com objetivo de promover ações que levam à inclusão na sociedade deste grupo de pessoas tão especiais, por meio da educação, do ingresso no mercado de trabalho, entre outras possibilidades.

A escolha do local se deu por conveniência, uma vez que a associação é parceira da instituição de ensino superior em atual graduação. $\mathrm{O}$ estudo foi realizado com duas crianças com diagnóstico de SD (média de crianças que apresentam critérios de inclusão para o atual estudo), com idade de 7 a 1218 anos, de ambos os sexos e etnia, residentes do município de Vitória da Conquista - BA. O critério de inclusão para participar da pesquisa foi: apresentar entendimento sobre o teste de manovacuometria e as solicitações para o desenvolvimento do treino psicomotor, após os pais ou responsáveis autorizarem a participação da criança no estudo. Os critérios de exclusão basearam-se no fato de além do diagnóstico de síndrome de Down, outros diagnósticos, como por exemplo: autismo ou transtorno de comportamento (TDAH).

No que se refere aos aspectos sociodemográficos, a primeira criança em estudo desse artigo possui 10 anos de idade, sexo feminino, branca, alfabetizada, realiza como atividades extracurriculares equoterapia, e tem acompanhamento da psicóloga e psicopedagoga. A respectiva genitora tem 48 anos, casada, branca, idade de gestação da criança 37 anos, ensino superior completo, residente da zona urbana de Vitória da Conquista.

Já a segunda criança em estudo desse artigo possui 06 anos, sexo masculino, pardo, não alfabetizado, acompanhado pela psicóloga e fonoaudióloga. A genitora tem 34 anos, solteira, parda, idade de gestação da criança 28 anos, ensino médio completo, residente da zona urbana de Vitória da Conquista.

Para responder os objetivos propostos no estudo foi utilizada na coleta de dados uma ficha roteiro, contendo dados sociodemográficos da mãe (iniciais do nome, data de nascimento, idade da gestação, renda familiar, etnia, naturalidade e local da residência); informações da criança participante do estudo (iniciais do nome, data de nascimento, etnia, sexo, escolaridade, atividades extracurriculares) e um campo que constou de data de avaliação e mensuração do manovacuômetro na primeira e última sessão de treino psicomotor pelas pesquisadoras da pesquisa.

576 Id on Line Rev. Mult. Psic. V.13, N. 48 p. 573-583, Dezembro/2019 - ISSN 1981-1179 Edição eletrônica em http://idonline.emnuvens.com.br/id 
Para verificar a capacidade respiratória das crianças participantes do estudo foi utilizado o manovacuômetro analógico, sendo este um instrumento rápido, não invasivo e indolor, que mensura a capacidade inspiratória máxima (Pimax) e a capacidade expiratória máxima (Pemax). Antes e depois de realizar os 10 treinamentos de circuito de psicomotricidade previamente programados, o circuito que foi montado possui um kit, que acompanha 06 estações, sendo: 47 bases, 08 semicírculos de tamanhos distintos, 04 aros, 12 balizas grandes, 11 balizas pequenas, 09 barreiras horizontais, 04 jogos de balizas com tamanhos diferenciados, 46 encaixes e 20 argolas de cores e tamanhos diferenciados.

O planejamento dos treinos psicomotores foi realizado com as duas crianças em mesmo horário, seguindo a programação: 10 minutos de aquecimento (com corrida, pular alto em cama elástica), 20 minutos com treinos de coordenação, equilíbrio, propriocepção, percepção corporal (saltar as barras em alturas diferentes, arrastar por baixo de meios arcos, passar por dentro de bambolês, fortalecimento de quadríceps e glúteos com caneleiras de $0,5 \mathrm{~kg}$ ), os 15 minutos finais do treino eram realizados com atividade psicomotora associado a tarefa (colocar os cones baixos em barras de apoio, pegar as bolas coloridas e anexar nos cones de cores similares), seguidos sempre de muita motivação para realização dos circuitos, assim como uma comemoração no final atribuindo a eles o título de campeão.

Vale ressaltar que esse estudo foi submetido ao Comitê de Ética em Pesquisa (CEP) da Faculdade Independente do Nordeste para apreciações. Depois de concedida a aprovação do estudo, através de número de parecer e número ??foi iniciada a coleta de dados, que aconteceu em duas etapas: a primeira etapa foi o contato com a coordenação da instituição, com agendamento prévio, para selecionar participantes do estudo segundo os critérios de inclusão do mesmo. Depois de selecionados os participantes, as mães ou responsáveis das crianças foram convidadas para uma reunião junto às pesquisadoras para apresentação dos objetivos do estudo.

Após a apresentação e esclarecimento das dúvidas, as mães que aceitaram participar do estudo, autorizaram o mesmo, com assinatura do Termo de Consentimento Livre e Esclarecido, impresso em duas cópias: uma para arquivamento do estudo e a outra foi entregue às mesmas. A segunda etapa foi realizada com a mensuração da capacidade respiratória das crianças selecionadas do estudo, em seguida foi realizado o agendamento prévio das sessões de intervenção do treino psicomotor (Kit de psicomotricidade e mobilidade infantil da marca physicus), que foi realizado na própria sede da associação. No total foram realizados 10 treinos psicomotores com cada criança, com o tempo estimado de 45 minutos de treino. No dia do 
último treino, foi realizada a reavaliação da capacidade respiratória das crianças através do manovacuômetro.

A análise dos dados se deu pela codificação através do Microsoft Excel 2013 e posteriormente realizada análise descritiva, foi utilizado o software estatístico SPSS (Statistical Package for the Social Sciences), versão 21.0.

\section{Resultados}

O estudo foi composto por duas crianças, uma do sexo feminino e um do sexo masculino, com idade de 10 e 6 anos respectivamente, onde foi possível avaliar a capacidade respiratória das crianças através do manovacuômetro antes e após a realização de dez sessões com treinos psicomotores estruturados. Segundo a comparação das médias das pressões inspiratórias e expiratórias, observou-se que as médias das pressões finais, inspiratórias e expiratórias aumentaram após o tratamento, contudo, não foi estatisticamente significativa a diferença dessas médias na pressão inspiratória (p-valor 0,266), entretanto com relação a pressão expiratória apresentou dados significativos ( $\mathrm{p}$-valor $=0,030)$, como apresentados na Tabela 1.

Tabela 1: Comparação das médias de pressões iniciais e finais, Vitória da Conquista, 2019

\begin{tabular}{lccc}
\hline & Média & Desvio Padrão & p-valor \\
\hline Pressão Inspiratória Inicial & 26,00 & 26,87 & 0,266 \\
Pressão Inspiratória Final & 35,00 & 21,21 & \\
\hline Pressão Expiratória Inicial & 8,50 & 2,12 & 0,030 \\
Pressão Expiratória Final & 40,00 & 0 & \\
\hline
\end{tabular}

Fonte: Dados da pesquisa. 2019.

\section{Discussão}

Considerando a fórmula apresentada por Schuster et al., (2012) como os valores previstos para a PEmáx e a PImáx em indivíduos saudáveis de todas as idades, os indivíduos deste estudo apresentam valores de PEmáx e PImáx abaixo do esperado para a faixa etária. Dado este que pode ser justificado pela amostra do estudo não ser realizado com crianças 
neuroatipicas. Em um estudo realizado em crianças com diagnóstico clínico de SD, Castoldiet al., (2012) avaliaram a força respiratória com manovacuômetro e observaram grave fraqueza muscular respiratória em todas as crianças, que posteriormente foram submetidas à intervenção com pressão de carga linear e apresentaram melhora significativa. Observou-se ainda que todos os sujeitos apresentavam fraqueza muscular, e os valores de PImáx e PEmáx embora menores que o previsto na literatura, foram superiores aos do estudo supracitado.

Modesto e Greguol (2014), em uma revisão bibliográfica com o objetivo de pesquisar valores de normalidade para pressões respiratórias máximas na faixa etária de 7 a 12 anos, concluíram que na maioria dos estudos, os sujeitos são avaliados na posição sentada com um clipe nasal. Os esforços máximos foram realizados a partir do volume residual e da capacidade pulmonar total, sustentada por um período de um a três segundos. Da mesma forma, Marinho (2018) recomenda a realização de três a cinco manobras para obter três manobras aceitáveis nas quais não houve vazamento. Desses três, dois devem ser reproduzíveis (com diferença inferior a $5 \%)$.

Neves et al., (2015) afirmam que os pacientes com SD apresentam uma série de características clínicas, principalmente hipotonia muscular. Este é um fator associado ao déficit de força muscular do tronco, que por sua vez resulta em perda de força muscular respiratória. Os mesmos autores avaliaram a função respiratória e criaram um perfil de crianças com SD entre 5 e 12 anos, revelando baixos valores de força muscular respiratória. Este estudo teve um resultado semelhante, em que os valores de PImáx e PEmáx de indivíduos de ambos os grupos estavam muito abaixo dos valores previstos para a idade.

Em um estudo que avaliou a força muscular respiratória em 45 indivíduos, dos quais 15 apresentavam deficiência intelectual, 15 apresentavam síndrome de Down e 15 foram classificados como sem deficiência mental, realizado por Orlando et al., (2014) mostraram que indivíduos com deficiência intelectual, com e sem síndrome de Down, apresentam menor PImáx e PEmáx, resultados estes que corroboraram com o atual estudo.Os valores da pressão expiratória máxima são maiores quando comparados aos da pressão inspiratória máxima em crianças de ambos os sexos. Isso também foi observado neste estudo, onde em ambos os grupos os valores médios dessas variáveis foram maiores para a PEmáx que a PImáx. Vale ressaltar que para Borssatt, Anjos e Ribas (2013), os valores mínimo e máximo para ambas as variáveis são respectivamente de 1 a 120 cmH ${ }_{2}$ Of, com valores negativos para a PImáx.

Silva e Valadares (2014) avaliaram PEmáx e PImáx. e observaram maior pressão respiratória máxima em meninos. Em um estudo com 40 crianças com idades entre 8 e 11 anos, 
Oliveira et al., (2014) demonstraram que o sexo influencia a força muscular respiratória em crianças e adolescentes. Eles mostraram que os meninos têm pressões mais altas que as meninas, devido ao fato de possuírem maior área muscular, embora, quando comparados aos adultos jovens do mesmo sexo, apresentem menor PEmáx, também devido ao menor desenvolvimento muscular.

Vale ressaltar que as fórmulas propostas para estimar a PImáx e a PEmáx, que têm como objetivo principal estabelecer rapidamente valores de referência nos testes de avaliação da força muscular respiratória, precisam ser observadas com cuidado. É o caso dos valores de PImáx e PEmáx previstos por Hasegawa et al., (2018), que é a única fórmula para valores previstos para toda a população brasileira. Os valores previstos de PImáx e PEmáx, estimados pela equação proposta por esses autores, para mulheres e homens, foram superiores aos obtidos nos resultados deste estudo.

A medida das pressões respiratórias máximas pode ajudar a direcionar objetivos terapêuticos, identificar doenças respiratórias precoces e classificar sua gravidade e avaliar respostas às terapias. As medidas das pressões inspiratórias e expiratórias máximas são determinantes na avaliação adequada da função pulmonar e podem ser realizadas em indivíduos saudáveis de diferentes idades ou em indivíduos com distúrbios e/ou diferentes tipos de alterações, como a síndrome de Down. Nesses pacientes, a medida sequencial das pressões respiratórias máximas permite quantificar a progressão da fraqueza muscular respiratória (ALSUBIE; ROSEN, 2017).

Castoldi et al., (2012) afirmam que indivíduos com SD apresentam déficit de força muscular respiratória e acreditam que esses indivíduos se beneficiam do treinamento muscular, o que minimiza futuras complicações respiratórias. De acordo com um estudo de Furlan et al., (2015), a incapacidade de manter a qualidade da postura e dos movimentos, característica comum às pessoas com SD, afeta o tronco e altera a harmonia da mecânica respiratória, modificando as pressões torácicas e abdominais. O desenvolvimento motor assistido equilibra o tórax e o abdômen, mantendo um ponto de equilíbrio entre essas duas cavidades e, assim, ajustando a capacidade e os volumes pulmonares

$\mathrm{O}$ treinamento de força em crianças com SD, de acordo com os dados atuais, poderia ser eficiente para melhorar a capacidade física durante atividades físicas, como caminhar. Isso é apoiado pela relação positiva entre força muscular e tolerância ao exercício por meio de análise de regressão múltipla. O treinamento físico é agora considerado um componente essencial da reabilitação pulmonar em pacientes com doença pulmonar obstrutiva crônica 
(DPOC), embora não altere as funções pulmonares, melhora a capacidade de exercício (BRAGA, et al., 2019).

Os resultados do presente estudo são corroborados pelo estudo de Zuchetto (2013) que mencionou que o estilo de vida sedentário de pessoas com SD é considerado um dos principais fatores que contribuem para a fraqueza muscular e hipotonia, que são as maiores prevalências de anormalidades circulatórias, má função do sistema pulmonar e diminuição dos níveis de aptidão física.

Os exercícios musculares aumentam a taxa e a profundidade da respiração que podem melhorar a CVF, além de aumentar o consumo de $\mathrm{O} 2$ e a taxa de difusão. Foi documentado por Alcântara (2018) que, durante o treinamento de resistência e força, o corpo exige mais oxigênio; portanto, os pulmões devem fornecer mais oxigênio aos músculos que trabalham através do sangue. À medida que a profundidade da respiração aumenta, a troca de oxigênio e dióxido de carbono entre os pulmões e o sangue ocorre de forma mais rápida e eficiente. Portanto, o exercício regular aumenta a capacidade pulmonar de fornecer oxigênio.

\section{Considerações Finais}

Os resultados deste estudo permitiram observar que a prática de treino psicomotor pode influenciar a força muscular respiratória em pacientes com síndrome de Down, de ambos os sexos, quando comparados com indivíduos neurotípicos que não praticam atividade física.

Identificamos como limitação desta pesquisa a pequena amostra, visto que houve dificuldade de entendimento nas crianças com SD para avaliação da força muscular respiratória PImáx e PEmáx, não sendo possível incluí-las. Diante dos resultados expostos, sugere-se então que novos estudos sejam realizados com uma maior amostra de indivíduos com Síndrome de Down, e que sejam inseridas na intervenção terapêuticas novas propostas de atividade funcional a serem realizadas.

\section{Referências}

ALCANTARA, A. D. O. Intervenção fisioterapêutica na sindrome de down. Unic, Cuiabá, p.130, 2018. 
ALSUBIE, H.S.; ROSEN, D. The evaluation and management of respiratory disease in children with Down Syndrome. Paediatr Respir. 2017.

BORSSATT, F.; ANJOS, F. B.; RIBAS, D. I. R.; Efeitos dos exercícios de força muscular na marcha de indivíduos portadores de Síndrome de Down. Fisioter. Mov, Curitiba, v. 26, n. 2, p.329-335, 17 jan. 2013.

BRAGA, H. V.; DUTRA, L. P.; VEIGA, J. M.; PINTO JUNIOR, E. P. Efeito da fisioterapia aquática na força muscular respiratória de crianças e adolescentes com Síndrome de Down. Arq. Cienc. Saúde UNIPAR, Umuarama, v. 23, n. 1, p, 9-13, jan./abr. 2019.

CASTOLDI, A. et al. Avaliação da força muscular e capacidade respiratória em pacientes com Síndrome de Down após Bad Ragaz. Revista Neurociências, São Paulo, v. 20, n. 3, p. 386-391, 2012.

FURLAN, F.R.; SGARIBOLDI, D.; BRIGATTO, P.; PAZZIANOTTO-FORTI, E.M. Avaliação da força muscular respiratória em indivíduos com síndrome de down. O Mundo da Saúde, São Paulo, v. 39, n. 2, p. 182-187, 2015.

HASEGAWA, J.; et al. Atuação da fisioterapia no desenvolvimento da criança com síndrome de down. Ciência Atual, Rio de Janeiro, v. 11, p.02-14, 2018.

MARINHO, M. F. S. A Intervenção fisioterapêutica no tratamento motor da síndrome de down. Campos do Saber, João Pessoa, v. 4, p.67-74, jun. 2018.

MODESTO, Everaldo; GREGUOL, Márcia. Influência do treinamento resistido em pessoas com Síndrome de Down-uma revisão sistemática. Revista Brasileira de Atividade Física \& Saúde, v. 19, n. 2, p. 153, 2014.

NEVES, Lilian Ferreira; DURAES, GrayceLaiz Lima Silveira; BEZERRA, Katia Francielly; CAPUCHINHO, Laura Caroline Ferreira Mende, Lopes Wanessa Casteluber. Hábitos alimentares: sua influencia no índice de massa corporal (IMC) em portadores de Síndrome de Down.Caderno de Ciências Agrárias, v7, n.2 p 40-44.2015.

OLIVEIRA, A.S.; GUERRA, R.R.S.; SOUZA A.T.; HOLANDA R.C.A.C.; SOARES F.O.. Estudo comparativo pneumofuncional entre crianças com síndrome de Down e não portadoras da síndrome. Rev Inspirar - movimento e saúde. v.6, n.4, p. 16-21, 2014.

ORLANDO, Camila Cherbaty da Silva; VIARO, Jacqueline Fernanda; FELIX, Pamela Ramalho; SIMÕES, Vivianne Augusta Pires. O desenvolvimento escolar da criança com Síndrome de Down. EDUCERE - Revista da Educação: Umuarama, v. 14, n. 2, p. 243-257, jul/dez. 2014.

RIBEIRO, Ricardo Nunes Rodrigues. A influência de um programa de exercício físico em meio terrestre na performance de nadadores de alto rendimento com Síndrome de Down. Porto. Dissertação de Mestrado apresentado a Faculdade de Desporto da Universidade do Porto. 2015. 
SCHUSTER, R.C.; ROSA, L.C.; FERREIRA, D.G.. Efeitos do treinamento muscular respiratório em pacientes com síndrome de down: estudo de casos. Rev Fisioter $S$ Fun. v. 1, pg $52-57,2012$.

SILVA, C. P.; VALADARES, Y. D. Avaliação da força muscular respiratória em indivíduos com Síndrome de Down. Conexão. UNIFOR-MG, Formiga, v. 9, n. 2, p. 24-37, jul./dez. 2014.

ZUCHETTO, Camila. Estado nutricional, consumo alimentar e atividade física de crianças e adolescentes com Síndrome de Down. Dissertação (Mestrado em Educação Física) - Escola Superior de Educação Física, Universidade Federal de Pelotas: Pelotas, 2013.

\section{Como citar este artigo (Formato ABNT):}

SILVA, Eduarda Stefany Silva e; DUTRA, Laisla Pires; PILOTO, Aline Marques; SARAIVA, Daiane Santos Dias. Avaliação Respiratória em Crianças com Síndrome de Down Submetidas a Treino Psicomotor . Id on Line Rev.Mult. Psic., Dezembro/2019, vol.13, n.48, p. 573-583. ISSN: 1981-1179.

Recebido: 23/11/2019

Aceito: 25/11/2019 